\section{International Journal of Medical \\ Research and Review}

MEDRESEARCH

www.medresearch.in

\title{
Open label case control prospective study to evaluate the impact of aceclofenac on antihypertensive action of amlodipine and ramipril in hypertensive patients with osteoarthritis
}

\author{
Babu Raman R. ${ }^{1}$, Khare A. ${ }^{2 *}$, Kumar D. ${ }^{3}$, Ghosh D. ${ }^{4}$, Tripathi N. ${ }^{5}$, Srivastava D. ${ }^{6}$, Prakash V. ${ }^{7}$, \\ Saurabh 5. ${ }^{8}$
}

DOI: https://doi.org/10.17511/ijmrr.2019.i01.08

${ }^{1}$ Ram Babu Raman, Tutor, ${ }^{2 *}$ A.K. Khare, Associate Professor, ${ }^{3}$ Deepak Kumar, Tutor, ${ }^{4}$ Dibyadeb Ghosh, The Head, ${ }^{\mathbf{5}}$ Neeraj Tripathi, Assistant Professor, Department of Medicine, 6 Deepak Srivastava, Assistant Professor, Department of Orthopaedics Surgery, Hind Institute of Medical Sciences, Barabanki, Uttar Pradesh, India.

7 Ved Prakash, Assistant Professor, ${ }^{\mathbf{8}}$ Seemant Saurabh, Assistant Professor; All the authors are affiliated with the Department of Pharmacology, Hind Institute of Medical Sciences, Barabanki, Uttar Pradesh, India.

\begin{abstract}
Objective: To study the impact of aceclofenac on antihypertensive action of amlodipine and ramiprilin hypertensive patients with osteoarthritis in an open label case control prospective study. Methods: This was an open label case control prospective study. Hypertensive patients on either amlodipine or Ramipril were included in control groups. A total of 120 patients were included in the study and divided into 4 groups: Group A- Hypertensivepatients on Ramipril; Group B- Hypertensive patients with concomitant osteoarthritis taking Aceclofenac and Ramipril; Group C- Hypertensive patients on Amlodipine and Group D- Hypertensive patients with concomitant osteoarthritis taking Aceclofenac and Amlodipine. Results: At the end of the first month (phase I) the ramipril subgroup in the control group had a mean systolic blood pressure of $136.73 \pm 3.80$ which was an $8.19 \%$ decrease from the baseline and it was found significant $(p<0.05)$. The systolic blood pressure measurements at the end of the second month (phase II) in the control groups revealed further fall in mean systolic blood pressure. The cases of osteoarthritis on aceclofenacand ramiprilshowedan increase in BP. The mean Systolic BP was $159.2 \pm 5.816$. An increase of $9.74 \%$ from the base line and $16.09 \%$ was noted at the end of phase-I $(P<0.5)$. Conclusion: The interaction of NSAIDs on the antihypertensive action of the ACE inhibitors is significantly greater than their blunting action on the calcium channel blockers.
\end{abstract}

Keywords: Hypertension, Antihypertensive drugs, ACE inhibitors

Corresponding Author

A.K. Khare, Associate Professor, Department of Pharmacology, Hind Institute of Medical Sciences, Barabanki, Uttar Pradesh, India.

Email: akharester@gmail.com

$\begin{array}{cc}\text { Manuscript Received } & \text { Review Round } \mathbf{1} \\ 2019-01-10 & 2019-01-20 \\ \text { Conflict of Interest } & \text { Funding } \\ \end{array}$

How to Cite this Article

Raman R, Khare AK, Kumar D, Ghosh D, Tripathi N, Srivastava D, Prakash V, Saurabh S. Open label case control prospective study to evaluate the impact of aceclofenac on antihypertensive action of amlodipine and ramipril in hypertensive patients with osteoarthritis. Int J Med Res Rev. 2019;7(1):43-49. Available From

https://ijmrr.medresearch.in/index.php/ijmrr/article/ view/1034

$$
\begin{gathered}
\text { Review Round } 2 \\
\text { 2019-01-26 } \\
\text { Ethical Approval } \\
\text { Yes }
\end{gathered}
$$

To Browse

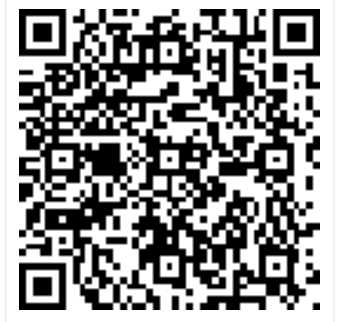
(c) 2019 by Ram Babu Raman, A.K. Khare, Deepak Kumar, Dibyadeb Ghosh, Neeraj Tripathi, Deepak Srivastava, Ved Prakash
Seemant Saurabh and Published by Siddharth Health Research and Social Welfare Society. This is an Open Access article licensed under a Creative Commons Attribution 4.0 International License https://creativecommons.org/licenses/by/4.0/ unported [CC BY 4.0].

Accepted 2019-01-31

Note 


\section{Introduction}

Given the high prevalence of hypertension, it is likely that concomitant use of NSAIDs and antihypertensive medication may occur in the same patient. There are numerous studies and metaanalyses that have focussed on the adverse effects of NSAIDs on blood pressure and the blunting of the efficacy of antihypertensive medications has been reported. Most classes of antihypertensive drugs appear to be affected; including diuretics, $\beta$ blockers, and angiotensin-converting enzyme inhibitors (ACEIs) [1-4].

\section{Aims \& Objective}

- To study the effect of aceclofenac on antihypertensive action of amlodipine in hypertensive patients with osteoarthritis.

- To study the effect of Aceclofenac on antihypertensive action of Ramipril in hypertensive patients with osteoarthritis.

- To compare the effect of Aceclofenac on antihypertensive action of Amlodipine and Ramipril in hypertensive patients with osteoarthritis.

\section{Methods}

Patients and Methods: The present study was an open label case control prospective study to evaluate the interaction between Aceclofenac and antihypertensive drugs Amlodipine and Ramipril in elderly patients.

Patient selection: A total of 120 patients aged between 40 to70 years of either sex attending our medicine and orthopaedic OPDs in the Hind Institute of Medical Science, Barabanki, U.P. were enrolled. Hypertensive patients on either amlodipine or ramipril were included in control groups. The study groups included hypertensive patients (taking ramipril or amlodipine) with concomitant osteoarthritis, requiring regular consumption of aceclofenac. An informed consent was obtained from all the enrolled patients. Patients above 70 of either sex or those presenting with medical or surgical complications were excluded. No surgical procedure was performed during the study. Any patient with history of intolerance or allergy to NSAIDs were also excluded.

Statistical Methods: ANOVA and Student's t-test of significancewas used for statistical analysis.

\section{Treatment Protocol}

Sample Size: A total number of 120 patients will be included after taking informed consent in the study.

The patients will be sorted in four groups and each group will contain 30 patients

Group A- Hypertensivepatients on Ramipril

Group B - Hypertensive patients with concomitant osteoarthritistaking Aceclofenacand Ramipril.

Group C- Hypertensive patients on Amlodipine

Group D- Hypertensive patients with concomitant osteoarthritis taking Aceclofenac and Amlodipine

Drug used in study: The drugs will be given to the patients on the basis of physician's discretion depending upon the hypertension and osteoarthritis

Aceclofenac: 100mgBD, Amlodipine: 5- $10 \mathrm{mg}$ OD, Ramipril: 5-20 mg OD/BD

A detailed history and thorough clinical examination will be done for each case. Specific emphasis will be given on the treatment and family history as well as life style modifications followed by the patients as instructed by the doctor.

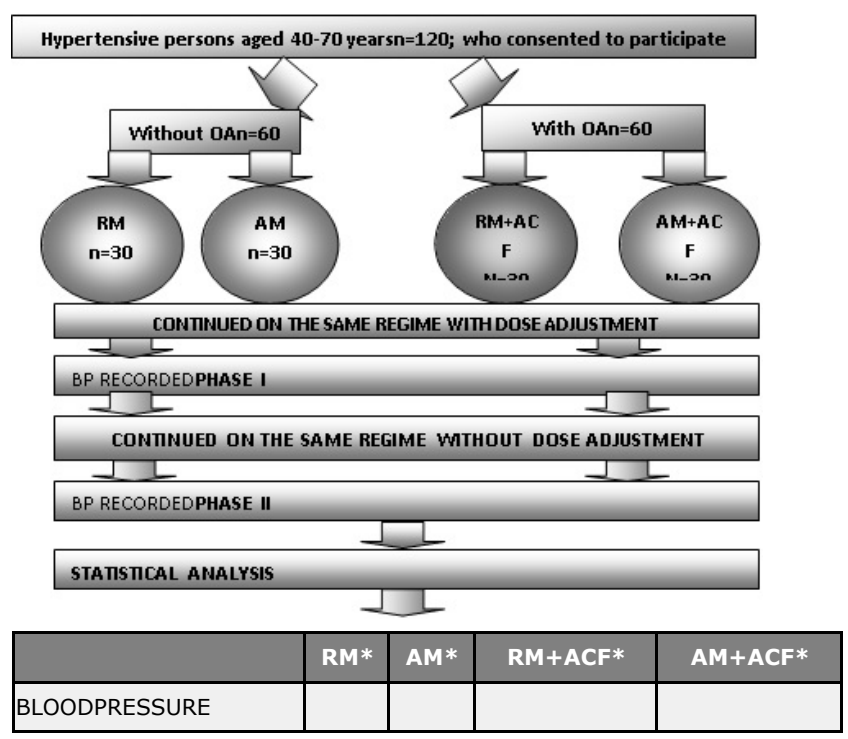

Flowchart Showing Study Inclusions and Exclusions

$* A M=$ Amlodipine $A C F=$ Aceclofenac;

$\mathrm{RM}=$ Ramipril.

NONE OF THE PATIENTS WERE LOST TO FOLLOW UP.

PROTOCOL FOR RECORDING OF BLOOD PRESSURE 
Recommended "Ideal" Cuff Sizes Adults

The most recent American Heart Association guidelines specify that the proper cuff has a bladder length of $80 \%$ and a width of at least $40 \%$ of arm circumferenceand determine the proper cuff size by determining the circumference of the arm at the half-way point between the olecranon and the acromion processes.

Arm Circumference (cm) Cuff Size (cm)

\begin{tabular}{|l|l|}
\hline 27 to 34 & $16 \times 30$ adult (standard) \\
\hline 35 to $4416 \times 36$ large adults \\
\hline 45 to 52 & $16 \times 42$ adult thighs \\
\hline
\end{tabular}

Equipment-the mercury sphygmomanometer remains the "gold standard" device for blood pressure measurement

Position of patient- The present consensus suggests that patients/clients should be seated quietly in a chair with back support, with both feet flat on the floor for at least 5 minutes prior to obtaining a measurement.

The initial visit blood pressure should be measured in both arms. According to Pickering et al "the patient should be instructed to relax as much as possible and to not talk during the measurement procedure." Blood pressure should also be measured in standing for those patients who are at risk for postural hypotension (e.g., elderly, patients with diabetes, and patients on antihypertensive medications).

\section{Results}

There were 120 patients; 61 males (50.83\%) and 59 females $(49.16 \%), 60$ of whom (50\%) were in the study groups and $60(50 \%)$ in the control groups. In the control group there were 25 women and 35 men, aged $57.67 \pm 4.348$ years, weighing $67.12 \pm 4.469 \mathrm{~kg}$ and their sitting Blood Pressure at the inception of this study (phase 0) was systolic $149.57 \pm 5.847$ (Table-1) and diastolic 89.27 \pm 4.715 .

There were 26 male hypertensive and 34 female hypertensive cases with concomitant osteoarthritis, the mean age group of the cases was 58.63 \pm 5.155 years, and it did not differ significantly from the control group (t test: $p>0.05$ ); the same was true with the weight of the cases $69.57 \pm 7.086 \mathrm{~kg}$ ( $t$ test: $p>0.05$ ).
Their sitting BP at the inception of the study was systolic $144.93 \pm 7.183$ and diastolic $88.90 \pm 3.282$ which also did not differ significantly (t test: $p>0.05$ ) from the control group. The average daily drug dosages were 5-10mg for ramipril, 5-10mg for amlodipine and $200 \mathrm{mg}$ for aceclofenac.

At the end of the first month (phase I) ramiprilsubgroup in the control group had a mean systolic blood pressure of $136.73 \pm 3.805$ which was an $8.19 \%$ decrease from the baseline and it was found significant ( $p$ value $<.05$ ) by one-way analysis of variance with post Bonferroni's Multiple Comparison Test. The same was true of the control group on amlodipine where the measured systolic blood pressure at the end of the first month of study was $135.6 \pm 4.133$ showingafall of $9.72 \%$ from the baseline. The above observation was also noted in the cases with osteoarthritis and on Aceclofenac. The Ramipril group on aceclofenac showed a mean systolic blood pressure of $137.13 \pm 5.625$. Thus, this group showed a fall of $5.47 \%$ which was statistically significant $(p<0.05)$.

The osteoarthritis patients on amlodipine and aceclofenac measured a mean systolic blood pressure of $138 \pm 3.063$, a $4.69 \%$ fall in BP from the baseline values and it was statistically significant $(p<0.05)$. All the results are tabulated (Table- 2)

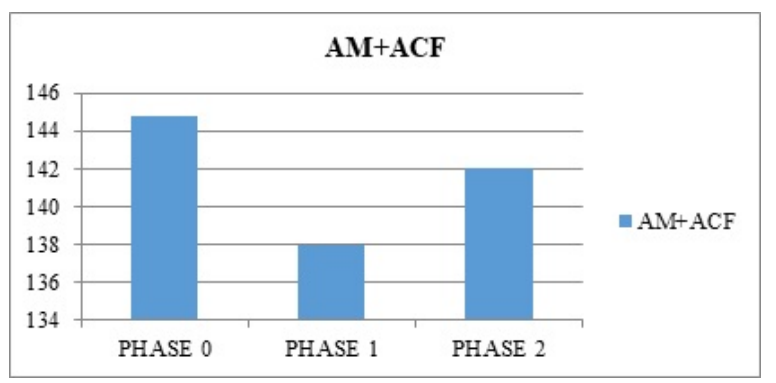

Table-1: Diastolic Blood Pressure Changes during the Study

\begin{tabular}{|c|c|c|c|c|}
\hline & RM & AM & RM+ACF & AM+ACF \\
\hline PHASE 0 & $87.87 \pm 4.334$ & $90.87 \pm 4.599$ & $89.00 \pm 3.742$ & $88.8 \pm 2.809$ \\
\hline PHASE 1 & $84.67 \pm 4.823$ & $85.87 \pm 4.133$ & $87.87 \pm 3.674$ & $85.6 \pm 2.699$ \\
\hline PHASE 2 & $81.67 \pm 3.407$ & $83.27 \pm 3.085$ & $94.00 \pm 2.626 *$ & $86.87 \pm 2.501$ \\
\hline
\end{tabular}

\section{$M E A N \pm S T A N D A R D$ DEVIATIONS OF DATA}

The diastolic blood pressure of the control group on ramipril recorded at the end of the first month showed a mean diastolic BP of $84.67 \pm 4.82$ about $3.64 \%$ fall from the baseline value but was statistically nonsignificant as found by Bonferroni's Multiple Comparison Test ( $p>0.05)$. 
The same was true with control group on Amlodipine which showed a mean diastolic BP of $85.87 \pm 4.133$, was considered statistically nonsignificant $(p>0.05)$.

The cases too showed a nonsignificant fall in mean diastolic BP at the end of the first month, the patients on ramipril and aceclofenac recorded a mean diastolic BP of $87.87 \pm 3.674(p>0.05)$. The other group on Amlodipine and Aceclofenac showed a mean diastolic BP of $85.60 \pm 2.699(p>0.05)$. The blood pressure recordings are tabulated below.

The blood pressure measurements at the end of the second month (phase II) in the control groups revealed further fall in both mean systolic and diastolic blood pressure. (Table-3,6) The Ramipril group patients had a mean systolic BP of $132.13 \pm 3.857$; a $11.28 \%$ from the baseline value (phase 0 ) and $3.6 \%$ fall from the phase 1 .

The decrease in the blood pressure from the baseline is significant $(p<0.05)$. With the amlodipine patients in the control group a similar significant fall in BP from the baseline values was seen, Bonferroni's multiple comparison test $(p<0.05)$ was obtained, the mean systolic BP was $130.67 \pm 3.644$, a $13.00 \%$ fall from the baseline value and $3.64 \%$ fall from phase $I$.

The cases of osteoarthritis on aceclofenac and ramipril showed an increase in $\mathrm{BP}$, the mean systolic blood pressure was $159.2 \pm 5.816$, a $9.74 \%$ increase from the baseline and $16.09 \%$ from the phase I. This is significant by the Analysis of variance post Bonferroni's Multiple Comparison Test $(p<0.05)$ when compared with phase 0 and $(p<0.05)$ against phase II.

Those osteoarthritis cases on aceclofenac and amlodipine were in contrast showed a fall in blood pressure from the baseline phase 0 . Their mean systolic blood pressure at the end of the second month phase II was $142.07 \pm 2.434$. About $1.88 \%$ fall from phase 0 which is significant $(p<0.05)$. But when compared to phase I BP, they showed a $2.94 \%$ increase in BP and it was found significant using the Analysis of variance post Bonferroni's Multiple Comparison Test $(p<0.05)$.

The diastolic blood pressure at the end of the second month in the control group showed a $7.59 \%$ decrease from the baseline value and a $3.6 \%$ from the phase $I$ in the ramipril group. The mean diastolic BP was $81.67 \pm 3.407$.
The analysis revealed the differences were statistically significant $(p<0.05)$ against phase 0 and $(p<0.05)$ against phase I. In the Amlodipine group the mean diastolic BP decreased by $8.36 \%$ from the baseline to a mean diastolic BP $83.27 \pm 3.084$ which was statistically significant $(p<0.05)$ and by $3.02 \%$ from the phase I but was not statistically significant (ANOVA: $p>0.05$.)

The cases of osteoarthritis on aceclofenac and ramipril showed a $5.61 \%$ increase in blood pressure from the baseline phase 0 value. The mean diastolic blood pressure was 94.00. The statistical significance was proved with Bonferroni's Multiple Comparison Test in one-way analysis of variance $(p<0.05)$. A $6.9 \%$ increase from the phase I diastolic BP was seen statistically significant (ANOVA: $p<0.05$ ). The amlodipine case group recorded a mean diastolic BP of $86.86 \pm 2.05$, a $2.18 \%$ decrease from the phase 0 this is statistically nonsignificant $(p>0.05)$. However, in comparison with the phase I they showed a $1.4 .7 \%$ increase in BP but this was not significant statistically $(p>0.05)$. The comparative values are tabulated below.

The mean body weight of all the patients at the end of the study was $68.34 \pm 6.026 \mathrm{~kg}$ and thus showed no considerable variation from the baseline value. Side effects in this study were minor and expected; e.g., three patients in the amlodipine study group and five in the amlodipine control group complained of some ankle edema but none withdrew from the trial.

\section{Discussion}

This study was meant to find the extent of the proven blunting effect of aceclofenac on some popularly used antihypertensive, namely amlodipine andramipril. Aceclofenac was selected because it is one of the most commonly prescribed, over-thecounter and freely available NSAID.

The present study was an open label, prospective control study. The design of the study was atypical since placebo control could not be done on account of technical and ethical reasons. However, this study had several advantages as well. It was performed in a busy hospital practice, highly resembling the reallife circumstances on a set of patients visiting our medicine and orthopedic out-patients regularly for the evaluation of their blood pressure and for their stock of analgesic in patients with osteoarthritis from the orthopedic. 
The study has confirmed the prohypertensive activity of aceclofenac known for decades [1-6]. The observed increase in blood pressure was much higher among the hypertensive cases in the present study (some $8-14 / 4-8 \mathrm{~mm} \mathrm{Hg}$ ) than in general populations (some $3-4 / 1-2 \mathrm{~mm} \mathrm{Hg}$ ). This outstanding worsening of pre-existent hypertension is clinically relevant, especially for elderly patients with comorbidity, exposed to additional cardiovascular risk factors (e.g. diabetes, nephropathy).

Blood pressure control during this study was remarkably good, achieving normotension in over $75 \%$ of the examinees at the end of the Phase I, but only $59 \%$ of the patients were normotensive at the end of the study in which over $50 \%$ of the normotensives were in the control group.

The data indicate that the blunting action of aceclofenac was evident on both antihypertensive drugs amlodipine and ramipril, but the effect on ramipril was proven statistically and was significant. This indicates that ACE inhibitors have greater tendency to lose their antihypertensive action when along given with NSAID like Aceclofenac.

This is in concordance with majority of the published trials $[7,8,9,10]$ but not with the study by Thakur et al [11] who found the antihypertensive effect of the combination of fosinopril and hydrochlorothiazide was resistant to the interference by NSAIDs. The present study confirmed the findings of the Conlin et al and Salvetti et al that the NSAID (indomethacin) blunts the antihypertensive effects of ACE inhibitors (captopril and losartan) [12]. Floor-Schreudering, et al (2015) [13] also observed interference in control of blood pressure in patients taking high dosage of new NSAID etoricoxib.

Turtle et al 14 observed that paracetamol, often suggested as a safer alternative to non-steroidal anti-inflammatory drugs is unlikely to interact with antihypertensive medications. They however suggested that further prospective evidence was needed to address the effect of paracetamol on BP.

Study earlier conducted by JM McKenney et al (1998) cited in reviewby Ruoff [15] had come to the conclusion from clinical trial data indicating that the blood pressure of patients with controlled hypertension can be raised by 3 to $6 \mathrm{~mm} \mathrm{Hg}$ during concurrent treatment with NSAIDs. The present study has used low dose aceclofenac for eight weeks, and that too on patients on antihypertensive therapy.
The blunting effect of aceclofenac, even at low doses but given for prolonged periods was clinically evident when used along with antihypertensive drugs in the present study.

The calcium channel blocker amlodipine seems to resist the untoward blunting action of aceclofenac and hence can be suggested a preferred antihypertensive over ACE inhibitor in patients who need NSAID for pain relief for long term relief from pain.

Since there was no significant increase in body weight of the patients during the study period, the elevation in BP hence could not be explained on the basis of volume expansion alone.

However, for understanding the complete mechanism and to prove the effect observed beyond doubt, a more scientific and methodical study over a larger population and for a longer period of time is necessary which is beyond the limitations of this study. Authors are contemplating to extend this study further and as data become available and are analyzed, they will be published.

\section{Conclusion}

The blunting effect of aceclofenac on some popularly used antihypertensive drugs such as amlodipine and ramipril was observed in some of the hypertensive patients suffering from osteoarthritis concomitantly.

One hundred twenty hypertensive patients were selected for the study and divided into two groups; those with osteoarthritis requiring Aceclofenac for the relief of pain as the study group and those without osteoarthritis as the control group.

The study group was further subdivided into 30 patients taking amlodipine and 30 patients already onramipril. Similarly, the control group also had two subgroups 30 patients on amlodipine and other 30 patients onramiprilbut not requiring NSAIDs.

After the baseline BP recordings (Phase 0 ), an attempt to control blood pressure by titrating the antihypertensive drug dosages was made in both the groups following which the blood pressures were again recorded after the period of 30 days (Phase I).

Both the groups were then followed for 30 days without dose adjustments; the control group was only on antihypertensive drugs while the study group was on antihypertensive as well as on Aceclofenac. 
The BP was again recorded at the end of the second month (Phase II). The body weight of all the subjects was also noted at Phase 0 and again at the end of Phase II. All results were then statistically analyzed.

This prospective open label case control study concludes the blunting effect of aceclofenac on antihypertensive drugs amlodipine and ramipril. It was observed that their blunting action of aceclofenac on ramipril was significant as when compared to aceclofenac blunting effect on amlodipine alone.

\section{Reference}

01. Wong DG, Spence JD, Lamki L, et al. Effect of non-steroidal anti-inflammatory drugs on control of hypertension by beta-blockers and diuretics. Lancet. 1986;327;997-1001.

[Crossref]

02. Beckmann ML, Gerber JG, Byyny RL, et al. Propranolol increases prostacyclin synthesis in patients with essential hypertension. Hypertension. 1988(6)582-8.

[Crossref]

03. Moore TJ, Crantz FR, Hollenberg NK, et al. Contribution of prostaglandins to the antihypertensive action of captopril in essential hypertension. Hypertension. 1981;3(2)168-73. [Crossref]

04. Quilley J, Duchin KL, Hudes EM, et al. The antihypertensive effect of captopril in essential hypertension- relationship to prostaglandins and the kallikrein-kinin system. J Hypertens. 1987;5(1)121-8.

[Crossref]

05. Chadha SL, Gopinath N, Shekhawat S. Urbanrural differences in the prevalence of coronary heart disease and its risk factors in Delhi. Bull World Health Organ. 1997;75(1)31-8.

[Crossref]

06. Gopinath N, Chadha SL, Jain P, et al. An epidemiological study of obesity in adults in the urban population of Delhi. J Assoc Physicians India. $1994 ; 42(3) 212-5$.

[Crossref]

07. Conlin PR, Moore TJ, Swartz SL. Effect of indomethacin on blood pressure lowering by captopril and losartan in hypertensive patients. Hypertension. 2000 Sep;36(3)461-5.

[Crossref]
08. Elizabeth A Chrischilles, Robert B. Wallace Nonsteroidal Anti-inflammatory Drugs and Blood Pressure in an Elderly Population- The Gerontological Society of America. Journal of Gerontology. 1993;48(3)M91-M96.

[Crossref]

09. Pavlicević I, Kuzmanić $M$, Rumboldt $M$, et al. Interaction between antihypertensives and NSAIDs in primary care- a controlled trial. Can J Clin Pharmacol. 2008;15(3)e372-82.

[Crossref]

10. Phillips BB, Joss JD, Mulhausen PL. Blood pressure elevation in a patient treated with salsalate. Ann Pharmacotherapy. 2002;4;624-7. [Crossref]

11. Thakur V, Cook ME, Wallin JD. Antihypertensive effect of the combination of fosinopril and HCTZ is resistant to interference by nonsteroidal antiinflammatory drugs. Am J Hypertens. 1999 Sep;12(9 Pt 1)925-8.

[Crossref]

12. Salvetti A, Abdel-Haq B, Magagna A, et al. Indomethacin reduces the antihypertensive action of enalapril. Clin Exp Hypertens $A$. 1987;9(2-3)559-67.

[Crossref]

13. Floor-Schreudering $A$, De Smet PA, Buurma $H$, et al. NSAID-antihypertensive drug interactionswhich outpatients are at risk for a rise in systolic blood pressure?. Eur J Prev Cardiol. 2015 (1)9195.

[Crossref]

14. Turtle EJ, Dear JW, Webb DJ. A systematic review of the effect of paracetamol on blood pressure in hypertensive and non-hypertensive subjects. $\mathrm{Br} \mathrm{J}$ Clin Pharmacol. 2013 Jun;75(6)1396-405.

[Crossref]

15. Ruoff GE. The impact of nonsteroidal antiinflammatory drugs on hypertension alternative analgesics for patients at risk. Clin Ther. 1998 May-Jun;20(3)376-87.

[Crossref] 